\title{
Antitumoral effects of attenuated Listeria monocytogenes in a genetically engineered mouse model of melanoma
}

\author{
Marianna Vitiello, ${ }^{1,2}$ Monica Evangelista ${ }^{1}$ - Nicole Di Lascio ${ }^{1}$ - Claudia Kusmic ${ }^{1}$ - Annamaria Massa ${ }^{3,4}$. \\ Francesca Orso ${ }^{3,4,5}$ - Samanta Sarti ${ }^{1,2}$ - Andrea Marranci ${ }^{1,2}$ - Katarzyna Rodzik ${ }^{6}$ - Lorenzo Germelli ${ }^{1,2}$. \\ Dinesh Chandra ${ }^{7}$ - Alessandra Salvetti ${ }^{8}$. Angela Pucci ${ }^{9} \cdot$ Daniela Taverna $^{3,4,5}$ - Francesco Faita ${ }^{1}$. \\ Claudia Gravekamp ${ }^{7} \cdot$ Laura Poliseno $\mathbb{1}^{1,2}$
}

Received: 3 July 2018 / Revised: 21 December 2018 / Accepted: 21 December 2018 / Published online: 21 January 2019

(c) The Author(s) 2019. This article is published with open access

\begin{abstract}
Attenuated Listeria monocytogenes $\left(\mathrm{Lm}^{\text {at }}\right.$-LLO) represents a valuable anticancer vaccine and drug delivery platform. Here we show that in vitro $\mathrm{Lm}^{\text {at }}$-LLO causes ROS production and, in turn, apoptotic killing of a wide variety of melanoma cells, irrespectively of their stage, mutational status, sensitivity to BRAF inhibitors or degree of stemness. We also show that, when administered in the therapeutic setting to Braf/Pten genetically engineered mice, Lm $^{\text {at }}$-LLO causes a strong decrease in the size and volume of primary melanoma tumors, as well as a reduction of the metastatic burden. At the molecular level, we confirm that the anti-melanoma activity exerted in vivo by $\mathrm{Lm}^{\text {at }}$-LLO depends also on its ability to potentiate the immune response of the organism against the infected tumor. Our data pave the way to the preclinical testing of listeria-based immunotherapeutic strategies against metastatic melanoma, using a genetically engineered mouse rather than xenograft models.
\end{abstract}

\section{Introduction}

Melanoma is the most aggressive form of skin cancer and remains a therapeutic challenge, especially when it reaches the metastatic stage [1]. Since one of the reasons behind aggressiveness resides in the marked ability displayed by melanoma cells to evade immune recognition, over the

Supplementary information The online version of this article (https:// doi.org/10.1038/s41388-019-0681-1) contains supplementary material, which is available to authorized users.

Marianna Vitiello

mariannavitiello@live.it

$\triangle$ Laura Poliseno

laura.poliseno@cnr.it

laura.poliseno@gmail.com

Institute of Clinical Physiology, CNR, Pisa, Italy

2 Oncogenomics Unit, Core Research Laboratory, ISPRO, Pisa, Italy

3 Molecular Biotechnology Center (MBC), University of Torino, Torino, Italy years many different therapeutic strategies have aimed at potentiating the immune response of the organism against the tumor. In particular, antibodies that unleash the full potential of helper and killer T-lymphocytes have already become a standard of care for metastatic melanoma patients [2], while the enhanced recognition of cancer neo-antigens holds great promise [3-5].

Spurred by these successful applications of systemic immunotherapy, we have decided to assess the antitumoral effects elicited by an attenuated and non-pathogenic version of Listeria monocytogenes $\left(\mathrm{Lm}^{\mathrm{a}}\right)$. Among the microorganisms exploited as anticancer vaccines, $\mathrm{Lm}^{\text {at }}$ was

4 Department of Molecular Biotechnology and Health Sciences, University of Torino, Torino, Italy

5 Center for Complex Systems in Molecular Biology and Medicine, University of Torino, Torino, Italy

6 Department of Biochemistry and Molecular Biology, Centre of Postgraduate Medical Education, Warsaw, Poland

7 Department of Microbiology and Immunology, Albert Einstein College of Medicine, New York, USA

8 Unit of Experimental Biology and Genetics, Department of Clinical and Experimental Medicine, University of Pisa, Pisa, Italy

9 Histopathology Department, Pisa University Hospital, Pisa, Italy 
a

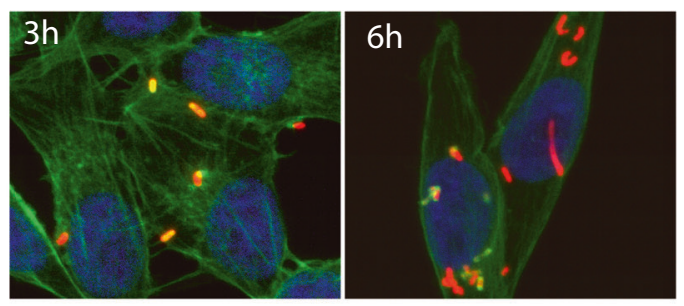

d

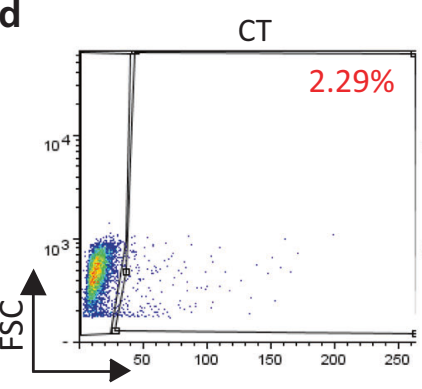

FL3
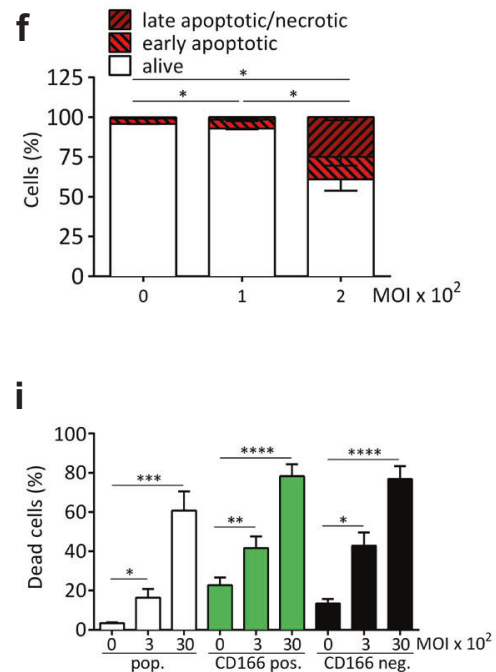

b

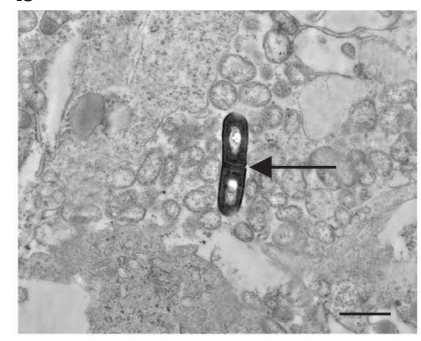

C

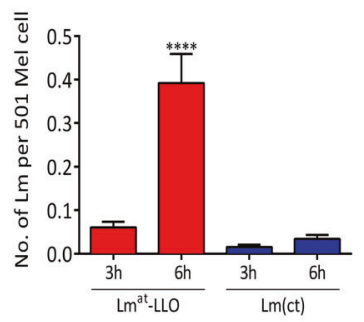

e

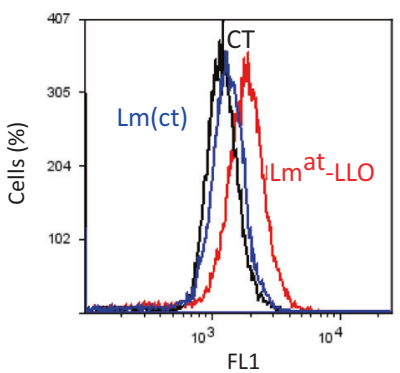

g

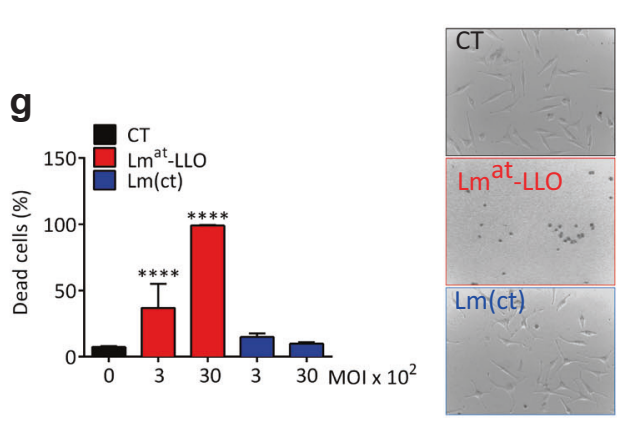

j

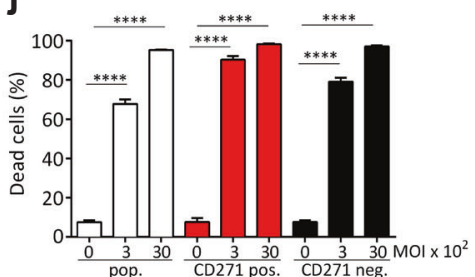

h

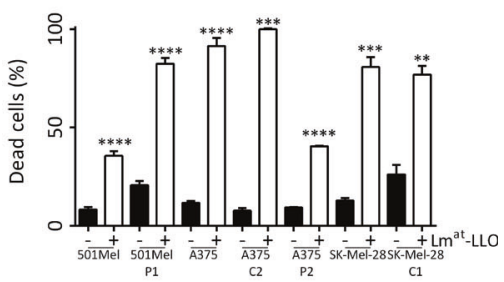

k

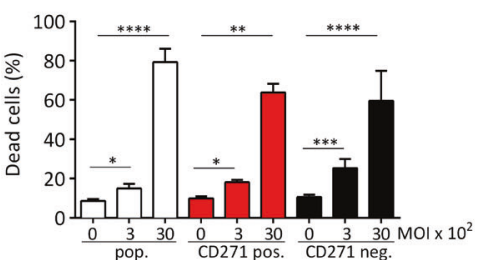

chosen in light of the strong and multifaceted immune response that it triggers [6,7], of its selective tropism for cancer cells [8], as well as of its tractability and versatility as drug delivery platform [9].

Here we show that the Lm $^{\text {at }}$-LLO strain [9] very efficiently kills a broad spectrum of melanoma cells in culture and, when injected in the therapeutic setting in a genetically engineered mouse model (GEMM) of melanoma [10], it greatly impairs the growth and metastatic burden of melanoma tumors.

\section{Results}

The ability of $\mathrm{Lm}^{\text {at }}$-LLO [9] (for a description of listeria strains used in this article, please refer to Supplementary
Fig. 1) to enter and replicate inside melanoma cells in culture was suggested by the immunofluorescent staining of intracellular clusters of bacteria (Fig. 1a) and was confirmed by transmission electron microscopy, since we captured dividing bacteria (Fig. 1b, Supplementary Fig. 2). Consistently, we detected an increase in $\mathrm{Lm}^{\text {at }}$-LLO infection rate over time (Fig. 1c). We also detected an increase in the number of listeria-positive melanoma cells, which indicates that $\mathrm{Lm}^{\text {at }}$-LLO is capable of spreading from cell to cell (Fig. 1d, Supplementary Fig. 2e). We then investigated whether $\mathrm{Lm}^{\text {at }}$-LLO infection results in cell mortality. Treating infected cells with CellROX reagent, we found that listeria causes the production of intracellular reactive oxygen species (ROS, Fig. 1e) [11]. This indeed results in apoptotic cell death (Fig. 1f, Supplementary Fig. 3), hence in a dramatic decrease in cell viability (Fig. 1g). Critically, 
4 Fig. 1 Lm $^{\text {at }}$-LLO infects and kills melanoma cells. a-c Lm ${ }^{\text {at }}$-LLO is able to replicate inside melanoma cells, as determined using immunofluorescence (a), electron microscopy (b), and infection rate (c). In a $501 \mathrm{Mel}$ cells were infected with MOI 3000 of Lm$^{\text {at }}$-LLO for $3 \mathrm{~h}$ (left panel) or $6 \mathrm{~h}$ (right panel). $\mathrm{Lm}^{\text {at }}$-LLO is stained in red using antiListeria antibody, the F-actin of tumour cells is stained in green using fluorescent Phallotoxin and the nuclei are stained in blue using DAPI. The red clusters indicate that $\mathrm{Lm}^{\text {at }}$-LLO is able to replicate in the cytoplasm of melanoma cells. Original magnification: $63 \times$. In b an example of listeria in division is captured using transmission electron microscopy. The black arrow indicates the septum that divides daughter cells (see Supplementary Fig. 2; scale bar: 500 nm). In c 501 Mel cells were infected with MOI 200 of $\mathrm{Lm}^{\text {at }}$-LLO. The increased infection rate when $3 \mathrm{~h}$ and $6 \mathrm{~h}$ are compared confirms that $\mathrm{Lm}^{\mathrm{at}}$-LLO is able to replicate inside melanoma cells. $\mathrm{Lm}(\mathrm{ct})$, which lacks LLO expression, is used as negative control. $\mathbf{d ~ L m}{ }^{\text {at }}$-LLO can spread across melanoma cells. $501 \mathrm{Mel}$ cells were infected with MOI $200 \mathrm{of} \mathrm{Lm}^{\text {at }}$ LLO for $3 \mathrm{~h}$ or $6 \mathrm{~h}$. Intracellular Lm ${ }^{\text {at }}$-LLO levels were evaluated using the anti-Listeria antibody. The increasing number of fluorescent cells indicates that $\mathrm{Lm}^{\text {at }}$-LLO is able to spread from cell to cell. Uninfected $501 \mathrm{Mel}$ cells are used as control (CT). e Infection of $501 \mathrm{Mel}$ cells with $\mathrm{Lm}^{\text {at }}$-LLO but not with $\mathrm{Lm}(\mathrm{ct})$ (MOI 200 for $6 \mathrm{~h}$ ) causes ROS production. $\mathbf{f}$ As a consequence of ROS production, an increase in AnnexinV-positive apoptotic cells is observed. $\mathrm{g}$ Kill rate of $\mathrm{Lm}^{\text {at }}$ LLO in $501 \mathrm{Mel}$ cells. Alive and dead cells were counted by trypan blue staining after $24 \mathrm{~h}$ of exposure to $\mathrm{Lm}^{\text {at }}$-LLO or Lm(ct) (MOI 300 and 3000). Representative pictures of CT, $\mathrm{Lm}^{\text {at }}$-LLO MOI 3000 and $\mathrm{Lm}(\mathrm{ct}) \mathrm{MOI} 3000$ are reported in the panels on the right. $\mathbf{h} \mathrm{Lm}^{\text {at }}$-LLO is effective at killing melanoma cells that show acquired resistance to vemurafenib. Kill rate in parental $501 \mathrm{Mel}$, A375 and SK-Mel-28 melanoma cells, as well as in their vemurafenib-resistant derivatives are reported. 501 Mel P1 resistant population and A375 C2 resistant clone are characterized by the expression of BRAFV600E $\Delta$ [3-10] splicing variant; in the A375 P2 resistant population, there is the KRAS K117N mutation; SK-Mel-28 C1 resistant clone is characterized by the overexpression of EGFR and PDGFRbeta [12]. Alive and dead cells were counted by trypan blue staining after $24 \mathrm{~h}$ of exposure to $\mathrm{Lm}^{\text {at }}$-LLO at MOI 3000 . $\mathbf{i}-\mathbf{k} \mathrm{Lm}^{\text {at }}$-LLO is effective at killing melanoma cells with different degree of stemness. i Kill rate on unsorted (pop), CD166 pos. and CD166 neg. SK-Mel-5 cells. j Kill rate on unsorted (pop), CD271 pos. and CD271 neg. SK-Mel-2 cells. k Kill rate on unsorted (pop), CD271 pos. and CD271 neg. SK-Mel-28 cells. Alive and dead cells were counted by trypan blue staining after $24 \mathrm{~h}$ of exposure to $\mathrm{Lm}^{\text {at }}$-LLO at MOI 3000. The graphs represent the mean \pm SEM of three independent experiments. $* p<0.05, * * p<0.01$, $* * * p<$ $0.001, * * * * p<0.0001$

none of the above-mentioned biological effects (replication inside melanoma cells (Fig. 1c), spreading across cells (Supplementary Fig. 4), ROS production (Fig. 1e) and cell killing (Fig. 1g)) was observed when we used the Lm(ct) strain, which is impaired in the expression of the bacterial protein LLO (Supplementary Fig. 1c). The different behavior displayed by $\mathrm{Lm}^{\text {at }}$-LLO and $\mathrm{Lm}(\mathrm{ct})$ attests that the biological effects observed with $\mathrm{Lm}^{\text {at }}$-LLO are consequences of the bacterial life cycle rather than of a general toxicity phenomenon.

Next, we aimed at assessing whether the ability of $\mathrm{Lm}^{\text {at }}$ LLO to kill melanoma cells is broad-spectrum. Indeed, we obtained similar kill rates in cell lines of different stage (primary and metastatic) and of different BRAF, NRAS, and NF1 mutational status (Supplementary Fig. 5). Interestingly, we also found that $\mathrm{Lm}^{\text {at }}$-LLO is effective at killing melanoma cells that are particularly refractory to treatment, such as those that display acquired resistance to vemurafenib due to different molecular mechanisms (Fig. 1h) [12] or those that are characterized by a higher degree of stemness (CD166-positive [13] SK-Mel-5 cells (Fig. 1i), CD271-positive [14] SK-Mel-2 and SK-Mel-28 cells (Fig. 1j-k, Supplementary Fig. 6).

Prompted by the strong effects observed in vitro, we moved on to the assessment of the antimelanoma activity exerted by $\mathrm{Lm}^{\text {at }}$-LLO in vivo. To this end, we exploited the tissue-specific and inducible GEMM of metastatic melanoma developed by Dankort and colleagues [10] (Supplementary Fig. 7). In this model, upon skin painting with 4hydroxitamoxifen (4-HT), Pten is loxed out (Supplementary Fig. 8a) and Braf acquires the V600E mutation, so that primary melanomas develop on site within few weeks (Supplementary Fig. 8b). These tumors are highly pigmented (i.e. the neoplastic cells are melanin rich), and heavily vascularized (Supplementary Fig. 8c-h). Furthermore, their growth is very fast-mice require euthanasia within 6-7 weeks after tumor induction-and metastasize rapidly to regional lymph nodes (LNs) and to the lungs (Supplementary Fig. 9-10).

In order to test the efficacy of $\mathrm{Lm}^{\text {at }}$-LLO in a therapeutic setting, we painted the back of 6 week-old mice with 4-HT and started $\mathrm{Lm}^{\text {at }}$-LLO injections 4 weeks later, when the primary tumor reached $50-100 \mathrm{~mm}^{3}$. One injection of high dose $\mathrm{Lm}^{\text {at }}$-LLO $\left(10^{7} \mathrm{CFU}\right)$ in the tumor area was followed by 14 intraperitoneal (IP) injections of a lower $\mathrm{Lm}^{\text {at }}$-LLO dose $\left(10^{5}\right.$ or $\left.10^{6} \mathrm{CFU}\right)$, one per day (Supplementary Fig. 11). The single intratumoral injection of a high dose of listeria favors the accumulation of the bacterium in the tumor microenvironment, while the serial injection of low doses stimulate the immune system to react against the bacterium itself [15]. The maximum dose of $\mathrm{Lm}^{\text {at }}$-LLO to be injected IP $\left(10^{6} \mathrm{CFU}\right)$ was chosen on the basis of IC50 calculation and absence of toxic side effects (Supplementary Fig. 12-13).

$\mathrm{Lm}^{\mathrm{at}}$-LLO treatment causes a conspicuous decrease in both the volume and the weight of primary tumors (Fig. 2a-c), so that the normal tissue, where the CRE-mediated deletion of Pten has not occurred, becomes more represented (Fig. 2d, left). Consistently with the results obtained in vitro, the impairment in tumor growth caused by $\mathrm{Lm}^{\text {at }}$-LLO is associated with an increase in the number of apoptotic Cleaved Caspase 3-positive cells (Fig. 2e). However, we also detected an increase in the levels of IL-2, a cytokine that promotes the differentiation of $\mathrm{T}$ cells (Fig. 2d, right) [16], as well as in the number of CD3+ T-lymphocytes recruited on site (Fig. 2f). Specifically, we detected a more pronounced infiltration of the CD4+ (Supplementary Fig. 14) 

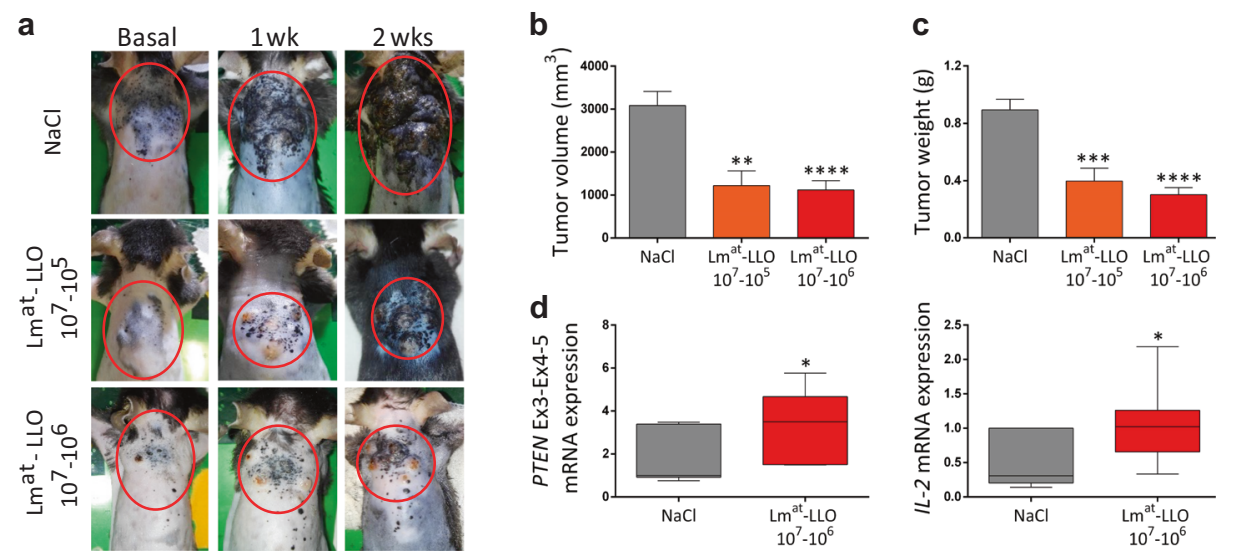

e
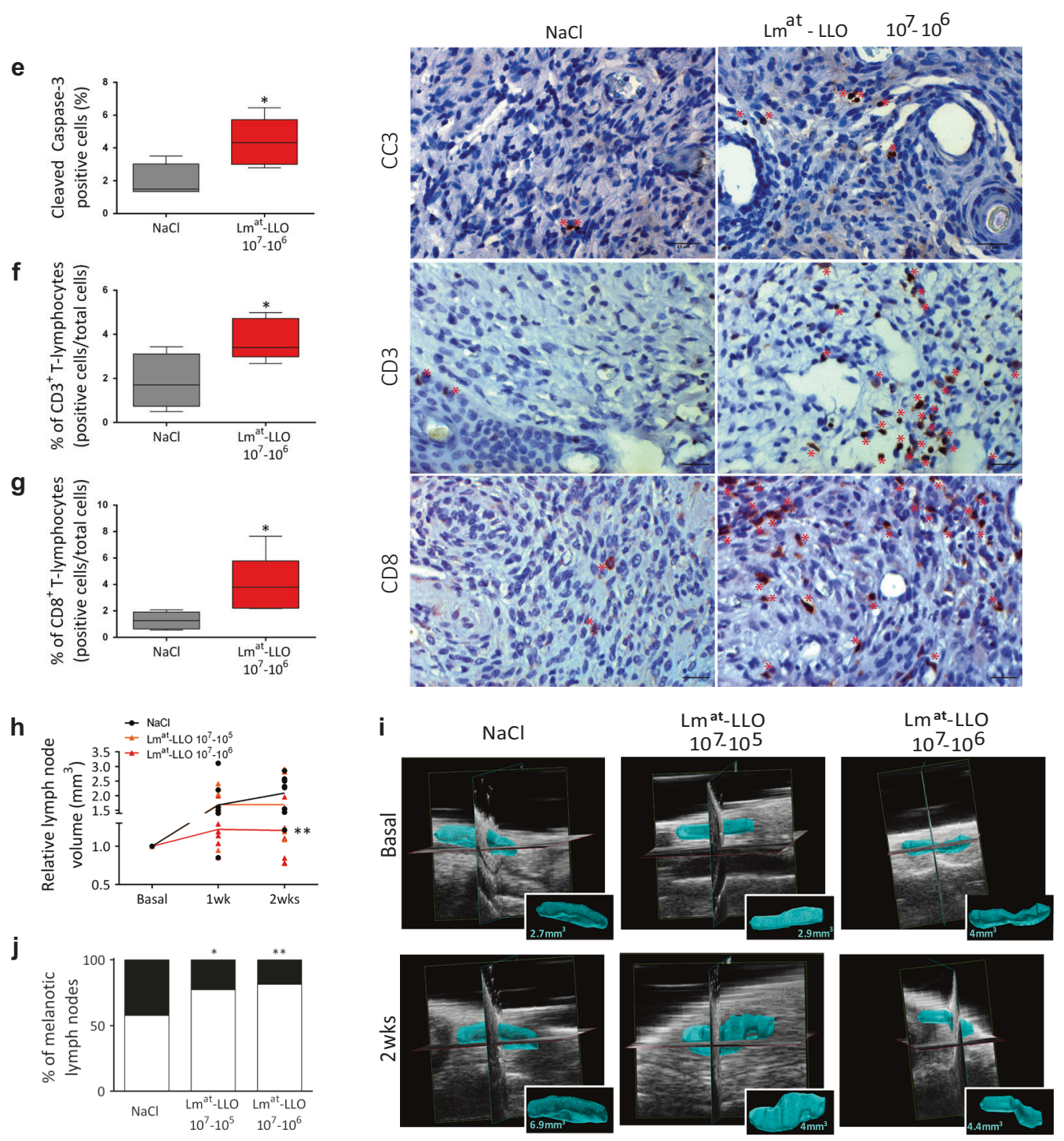

and the CD8+ (Fig. 2g) subpopulations, which are responsible for cell-mediated and cytotoxic immunity against listeria, respectively [7, 15, 17]. These results confirm that in vivo $\mathrm{Lm}^{\text {at }}$-LLO potentiates the response of the immune system against the tumor and, therefore, that its antitumoral effects are at least in part non-cell autonomous [18].

We also assessed the ability of Lm ${ }^{\text {at }}$-LLO treatment to impair the metastatic burden. Indeed, the measurements obtained by ultrasound imaging indicate a decrease in the 
Fig. $2 \mathrm{Lm}^{\text {at }}$-LLO inhibits the growth of primary melanoma tumors and their metastatization to lymph nodes. a-c $\mathrm{Lm}^{\text {at }}$-LLO inhibits the growth of primary melanoma tumors. a Representative pictures of primary tumors (red line) developed by mice treated with saline solution $(\mathrm{NaCl})$ or the indicated doses of $\mathrm{Lm}^{\text {at }}$-LLO (one injection of $10^{7} \mathrm{CFU}$ in the tumor area was followed by one IP injection per day of $10^{5}$ or $10^{6} \mathrm{CFU}$ for 14 days). For each experimental group, pictures were taken at three time points (before treatment, after 1 week of treatment and after 2 weeks of treatment). b, c Volume (b) and weight (c) of primary tumors. The number of primary tumors (mice) analyzed are: 12 (volume) and 13 (weight) for the $\mathrm{NaCl}$ group, 7 (volume) and 8 (weight) for the $\mathrm{Lm}^{\text {at }}$-LLO $10^{7}-10^{5}$ group and 12 (volume and weight) for the $\mathrm{Lm}^{\text {at }}$-LLO $10^{7}-10^{6}$ group. d Expression levels of undeleted Pten mRNA and $I L-2$ mRNA. Total RNA extracted from paraffin embedded primary tumor samples was analyzed by qRT-PCR. Left: Undeleted Pten mRNA levels were measured using a forward primer located on exon 3 and a reverse located on exon 4-5, as reported in [26]. The higher levels of undeleted Pten mRNA detected in mice treated with $\mathrm{Lm}^{\text {at }}$-LLO compared to control mice are consistent with the smaller size of primary tumors. Right: The higher levels of $I L-2$ mRNA detected in $\mathrm{Lm}^{\text {at }}$-LLO treated mice compared to control mice provide a molecular confirmation of the induction of the immune system by the vaccine. e Infection of tumor cells with $\mathrm{Lm}^{\text {at }}$-LLO causes a significant increase in apoptotic cell death, as indicated by Cleaved Caspase- 3 immunostaining. The number of primary tumors (mice) analyzed is five for each experimental group. Original magnification: $40 \times$ (scale bar: $25 \mu \mathrm{m}$ ). $\mathbf{f}$, $\mathbf{g}$ Infection of tumor cells with $\mathrm{Lm}^{\text {at }}$ LLO causes a significant increase in T-lymphocytes infiltration, as indicated by immunostaining of $\mathrm{CD} 3+(\mathbf{f})$ and $\mathrm{CD} 8+(\mathbf{g})$ cells. The number of primary tumors (mice) analyzed is five for each experimental group. Original magnification: $40 \times$ (scale bar: $20 \mu \mathrm{m}$ ). In b-g the mean \pm SEM is reported. $* p<0.05$, **p $p<0.01$, ***p $p<0.001$, $* * * * p<0.0001 . \mathbf{h}, \mathbf{i ~ L m}{ }^{\text {at }}$-LLO inhibits melanoma metastatization to lymph nodes. $\mathbf{h}$ Volume of inguinal lymph nodes in mice treated with saline solution $(\mathrm{NaCl})$ or the indicated doses of $\mathrm{Lm}^{\text {at }}$-LLO, as measured by ultrasound imaging. The volume of right and left inguinal lymph nodes was measured at three time points (before treatment, after 1 week and after 2 weeks of treatment). The total number of lymph nodes (mice) analyzed was eight (four) for each experimental group. In the graph, individual measurements are reported, as well as a line connecting mean values. ${ }^{* *} p<0.01$. i $3 \mathrm{D}$ ultra high-frequency ultrasound reconstruction of a representative inguinal lymph node per experimental group (with surface rendering and volume measurement reported as picture in picture). The upper images were acquired before treatment, while the lower images were acquired after 2 weeks of treatment. j Melanin content of axillary, brachial, and inguinal lymph nodes in mice treated with saline solution $(\mathrm{NaCl})$ or the indicated doses of $\mathrm{Lm}^{\text {at }}$-LLO. The total number of lymph nodes (mice) analyzed was the following: 59 (13) for the $\mathrm{NaCl}$ group, 44 (8) for the $\mathrm{Lm}^{\text {at }}$ LLO $10^{7}-10^{5}$ group, 59 (12) for the Lm ${ }^{\text {at }}$-LLO $10^{7}-10^{6}$ group. Black, high melanin content; white, no or low melanin content. $* p<0.05$, $* * p<0.01$

volume of regional LN (Fig. 2h, i and Supplementary Fig. 15), which, according to visual inspection, correlates with a decrease in melanin/melanoma deposits (Fig. 2j). Consistently, we observed a decrease in the number of metastatic nodules in the lungs (Supplementary Fig. 16).

$\mathrm{Lm}^{\text {at }}$-LLO treatment was not accompanied by overall toxicity-it didn't cause weight loss or liver lesions (Supplementary Fig. 17) - and was characterized by selective accumulation of bacteria at the anatomic sites of tumor growth, confirming the renowned tropism of listeria for cancer cells (Supplementary Fig. 18).

\section{Discussion}

The data we obtained in vitro attest the ability of $\mathrm{Lm}^{\text {at }}$-LLO to kill a wide variety of melanoma cells. Therefore, they suggest that $\mathrm{Lm}^{\text {at }}$-LLO treatment could overcome one of the most challenging features displayed by melanoma tumors, namely their high degree of heterogeneity [19].

Even more importantly, the data obtained in Braf/Pten mice reveal for the first time that the activity of $\mathrm{Lm}^{\text {at }}$-LLO is strong enough to be observed in spite of the fact that the melanoma tumors, which form in a GEMM, are much more heterogeneous than those that form in the xenograft models used thus far [20]. Therefore, they offer the rationale for testing new first-line treatment strategies that revolve around $\mathrm{Lm}^{\text {at }}$-LLO and for doing so in a preclinical setting that resembles human melanoma more closely, hence is endowed with higher translational relevance.

Considering its high versatility as a drug carrier, we suggest that $\mathrm{Lm}^{\text {at }}$-LLO could be engineered to express melanoma-associated antigens [21], so that the immune system of the body is boosted even further, or conjugated with a BRAF or a MEK inhibitor, providing an alternative way to assess the combined effects of immunotherapy and targeted therapy [22].

In light of the results we obtained in vitro, it should be considered that injections of $\mathrm{Lm}^{\text {at }}$-LLO might be effective also as a second-line treatment, on Braf/Pten mice that have become resistant to BRAF [23] or MEK [10] inhibitors.

Finally, Braf/Pten mice can be used to evaluate if the effects of $\mathrm{Lm}^{\text {at }}$-LLO are durable in time and if the tumors that eventually start growing again remain sensitive to a rechallenge [20]. Moreover, if mice with the Tyr::CreER+, BrafCA/+, Pten+/lox genotype instead of the Tyr::CreER + , BrafCA/+, Ptenlox/lox genotype are used for the induction, primary tumors grow more slowly and morbidity becomes related to metastases [24]. Therefore, a more thorough assessment of the effects of $\mathrm{Lm}^{\text {at }}$-LLO on metastatic sites can be obtained.

\section{Materials and methods}

\section{Strains of Listeria monocytogenes}

The characteristics of the strains of Listeria monocytogenes used in this work ( $\mathrm{Lm}^{\text {at }}$-LLO and $\left.\mathrm{Lm}(\mathrm{ct})\right)$ are described in Supplementary Fig. 1. 
To obtain $\mathrm{Lm}^{\text {at }}$-LLO, the XFL-7 strain was electroporated with pGG-34 plasmid as reported in [25].

Analogously, to obtain Lm(ct), the XFL-7 strain was electroporated with the pGG-34-OVA ${ }_{214-386}$ plasmid. Both strains were subsequently grown in BHI medium with 34 $\mu \mathrm{g} / \mathrm{ml}$ chloramphenicol at $37^{\circ} \mathrm{C}$.

\section{Mice strain}

B6.Cg-Braf ${ }^{\mathrm{tm} 1 \mathrm{Mmcm}} \quad \operatorname{Pten}^{\mathrm{tm} 1 \mathrm{Hwu}} \operatorname{Tg}($ Tyr-cre/ERT2)13Bos/ BosJ mice were purchased from the Jackson Laboratory (013590). For in vivo experiments, Tyr::CreER+ (heterozygous for CreER), BrafCA/+ (heterozygous for BrafV600E), and Ptenlox/lox (homozygous for Pten loss) mice were used, as described in Supplementary Fig. 11. Mice were treated in compliance with the animal protocol \#754/2015-PR, initially approved by the Italian Ministry of Heath on July 27th 2015 and then amended on September 12th 2018.

\section{Statistical analyses}

Statistical analyses were performed using unpaired and twotailed Student $t$-test or Chi-square test, as needed. Values of $p<0.05$ were considered statistically significant $\left({ }^{*} p<0.05\right.$, $* * p<0.01, * * * p<0.001, * * * * p<0.0001)$.

Acknowledgements The authors would like to thank all the Poliseno lab members for their insightful discussions; S. Burchielli (FTGM, Pisa) for her assistance with animal procedures; D. Colecchia (IFCCNR, ISPRO, Siena) for his assistance in preparing the figures; M. La Ferla (Fondazione Pisana per la Scienza, Pisa) for his assistance with the detection of listeria by FACS; G. Pelosi (IFC-CNR, Pisa) for his assistance with light microscopy; A. Ripoli (IFC-CNR, Pisa) for his support with statistical analyses; B. Stecca (ISPRO, Firenze) for providing us with the Aldefluor ${ }^{\mathrm{TM}}$ kit. This work was supported by MFAG \#17095 grant awarded by Associazione Italiana per la Ricerca sul Cancro (AIRC) to LP; start up funding awarded by ITT-Istituto Toscano Tumori and ISPRO-Istituto per lo Studio, la Prevenzione e la Rete Oncologica to LP. It was also supported by GR-2011-02348535 grant awarded by Italian Ministry of Health to LP. MV was supported by an EMBO short-term fellowship and an AIRC fellowship.

Author contributions LP conceived the project and supervised the research; MV and LP designed the experiments; MV, ME, NDL, CK, AM, FO, SS, AndM, KR, LG, DC, AS, and LP performed the experiments; MV, CK, FO, AS, AP, DT, FF, CG, and LP analyzed the data; LP and MV wrote the manuscript, with the input of all authors. All authors discussed and approved the manuscript.

\section{Compliance with ethical standards}

Conflict of interest The authors declare that they have no conflict of interest.

Publisher's note: Springer Nature remains neutral with regard to jurisdictional claims in published maps and institutional affiliations.
Open Access This article is licensed under a Creative Commons Attribution 4.0 International License, which permits use, sharing, adaptation, distribution and reproduction in any medium or format, as long as you give appropriate credit to the original author(s) and the source, provide a link to the Creative Commons license, and indicate if changes were made. The images or other third party material in this article are included in the article's Creative Commons license, unless indicated otherwise in a credit line to the material. If material is not included in the article's Creative Commons license and your intended use is not permitted by statutory regulation or exceeds the permitted use, you will need to obtain permission directly from the copyright holder. To view a copy of this license, visit http://creativecommons. org/licenses/by/4.0/.

\section{References}

1. Menzies AM, Long GV. Recent developments in melanoma therapy. JAMA Oncol. 2016;2:1259-60.

2. Kee D, McArthur G. Immunotherapy of melanoma. Eur J Surg Oncol. 2017;43:594-603.

3. Sahin U, Derhovanessian E, Miller M, Kloke BP, Simon P, Lower $\mathrm{M}$, et al. Personalized RNA mutanome vaccines mobilize polyspecific therapeutic immunity against cancer. Nature. 2017;547:222-6.

4. Ott PA, Hu Z, Keskin DB, Shukla SA, Sun J, Bozym DJ, et al. An immunogenic personal neoantigen vaccine for patients with melanoma. Nature. 2017;547:217-21.

5. Hellmann MD, Snyder A. Making it personal: neoantigen vaccines in metastatic melanoma. Immunity. 2017;47:221-3.

6. Wood LM, Paterson Y. Attenuated Listeria monocytogenes: a powerful and versatile vector for the future of tumor immunotherapy. Front Cell Infect Microbiol. 2014;4:51.

7. Flickinger JC, Jr., Rodeck U, Snook AE. Listeria monocytogenes as a vector for cancer immunotherapy: current understanding and progress. Vaccines (Basel) 2018; 6:3.

8. Quispe-Tintaya W, Chandra D, Jahangir A, Harris M, Casadevall A, Dadachova E, et al. Nontoxic radioactive Listeria(at) is a highly effective therapy against metastatic pancreatic cancer. Proc Natl Acad Sci USA. 2013;110:8668-73.

9. Chandra D, Selvanesan BC, Yuan Z, Libutti SK, Koba W, Beck A, et al. 32-Phosphorus selectively delivered by listeria to pancreatic cancer demonstrates a strong therapeutic effect. Oncotarget. 2017;8:20729-40.

10. Dankort D, Curley DP, Cartlidge RA, Nelson B, Karnezis AN, Damsky WE Jr., et al. Braf(V600E) cooperates with Pten loss to induce metastatic melanoma. Nat Genet. 2009;41:544-52.

11. Kim SH, Castro F, Paterson Y, Gravekamp C. High efficacy of a Listeria-based vaccine against metastatic breast cancer reveals a dual mode of action. Cancer Res. 2009;69:5860-6.

12. Vitiello M, Tuccoli A, D'Aurizio R, Sarti S, Giannecchini L, Lubrano S, et al. Context-dependent miR-204 and miR-211 affect the biological properties of amelanotic and melanotic melanoma cells. Oncotarget. 2017;8:25395-417.

13. Klein WM, Wu BP, Zhao S, Wu H, Klein-Szanto AJ, Tahan SR. Increased expression of stem cell markers in malignant melanoma. Mod Pathol. 2007;20:102-7.

14. Boiko AD, Razorenova OV, van de Rijn M, Swetter SM, Johnson DL, Ly DP, et al. Human melanoma-initiating cells express neural crest nerve growth factor receptor CD271. Nature. 2010;466:133-7.

15. Jahangir A, Chandra D, Quispe-Tintaya W, Singh M, Selvanesan BC, Gravekamp C. Immunotherapy with Listeria reduces metastatic breast cancer in young and old mice through different mechanisms. Oncoimmunology. 2017;6:e1342025. 
16. Liao W, Lin JX, Leonard WJ. IL-2 family cytokines: new insights into the complex roles of IL-2 as a broad regulator of T helper cell differentiation. Curr Opin Immunol. 2011;23:598-604.

17. Deng W, Lira V, Hudson TE, Lemmens EE, Hanson WG, Flores $\mathrm{R}$, et al. Recombinant Listeria promotes tumor rejection by CD8 (+) T cell-dependent remodeling of the tumor microenvironment. Proc Natl Acad Sci USA. 2018;115:8179-84.

18. Pan ZK, Weiskirch LM, Paterson Y. Regression of established B16F10 melanoma with a recombinant Listeria monocytogenes vaccine. Cancer Res. 1999;59:5264-9.

19. Grzywa TM, Paskal W, Wlodarski PK. Intratumor and intertumor heterogeneity in melanoma. Transl Oncol. 2017;10:956-75.

20. Xin G, Schauder DM, Jing W, Jiang A, Joshi NS, Johnson B, et al. Pathogen boosted adoptive cell transfer immunotherapy to treat solid tumors. Proc Natl Acad Sci USA. 2017;114:740-5.

21. Buonaguro L, Petrizzo A, Tornesello ML, Buonaguro FM. Translating tumor antigens into cancer vaccines. Clin Vaccin Immunol. 2011;18:23-34.
22. Karachaliou N, Gonzalez-Cao M, Sosa A, Berenguer J, Bracht JWP, Ito M, et al. The combination of checkpoint immunotherapy and targeted therapy in cancer. Ann Transl Med. 2017;5:388.

23. Yuan P, Ito K, Perez-Lorenzo R, Del Guzzo C, Lee JH, Shen CH, et al. Phenformin enhances the therapeutic benefit of BRAF (V600E) inhibition in melanoma. Proc Natl Acad Sci USA. 2013;110:18226-31.

24. Damsky WE, Curley DP, Santhanakrishnan M, Rosenbaum LE, Platt JT, Gould Rothberg BE, et al. Beta-catenin signaling controls metastasis in Braf-activated Pten-deficient melanomas. Cancer Cell. 2011;20:741-54.

25. Kim SH, Castro F, Gonzalez D, Maciag PC, Paterson Y, Gravekamp C. Mage-b vaccine delivered by recombinant Listeria monocytogenes is highly effective against breast cancer metastases. Br J Cancer. 2008;99:741-9.

26. Trotman LC, Niki M, Dotan ZA, Koutcher JA, Di Cristofano A, Xiao A, et al. Pten dose dictates cancer progression in the prostate. PLoS Biol. 2003;1:E59. 